\title{
Circular RNAs in plants
}

\author{
C. Ye, Q. Chu, L. Chen, X. Zhang, L. Fan* \\ Institute of Crop Science and Institute of Bioinformatics, Zhejiang University, Hangzhou, China \\ *e-mail:fanlj@zju.edu.cn
}

Key words: plant genomics, circular RNA, Oryza sativa, Arabidopsis thaliana, splicing, database

Circular RNAs (circRNAs) are covalently closed loops derived from back-splicing of precursor mRNAs. Recently, a large number of circRNAs have been detected among diverse organisms. For the first time in plants, we have identified 12,037 and 6,012 circRNAs in Oryza sativa and Arabidopsis thaliana, respectively. We demonstrated that circRNAs are widespread in plants and revealed the common and distinct features of circRNAs between plants and animals. We found that there are a certain number of circRNAs with sequence and splicing position conservation in plants, demonstrating the important role of circRNAs in plant growth, development and evolution. Regarding to the novelty of plant genomes/genes, a software for prediction of plant circRNAs, termed PcircRNA_finder, which is more sensitive and precise in detecting plant circRNAs than other frequently used programs. Another software, termed circseq-cup, was also developed, which can assemble the full-length sequences of circRNAs using the back-splicing RNA-Seq reads and their corresponding paired-end reads. We for the first time identified full-length sequences of nearly 3,000 circRNAs from $O$. sativa genome. Based on the full-length sequences, we showed that alternative circularization of circRNA is a common feature in $O$. sativa and, surprisingly, found that the junction sites of a large number of circRNAs are flanked by diverse non-GT/AG splicing signals in $O$. sativa. Moreover, we have created a database of plant circRNAs, PlantcircBase (http://ibi.zju.edu.cn/plantcircbase/). We have collected publicly available circRNAs identified in recent years by bioinformatics prediction and/or experimental validation, as well as circRNAs newly identified by our own lab in rice and Arabidopsis. Apart from the detailed and comprehensive information for each circRNA entry, PlantcircBase also provides basic tools including browsing, searching and downloading as well as advanced tools, including visualizing structures of circRNAs and predicting circRNAs. Part of our results have been published in Ye et al., 2015, New Phytologist; Chen et al., Bioinformatics; Ye et al., 2017, RNA Biology; Chu et al., 2017, Molecular Plant. 